\title{
Empfiehlt es sich, einen Alternativen Juristentag einzurichten?:
}

Das altfränkische Einleitungswort »empfiehlt« spielt auf die rituelle Formel an, mit der der Deutsche Juristentag (DJT) seit 19 ro das Beratungsthema für eine Abteilung vorstellt. Diese Formel hatte auch der Referent gewählt, als er 1970 in der Kritischen Justiz vorschlug, ${ }^{1}$ den Juristentag überhaupt abzuschaffen - an einen "Alternativen Juristentag " hatte er noch nicht gedacht. Hier wird schon von "Heerschauen konservativer Juristen * von »Bekenntnistagen für das abgestandene Ordnungsbild bürgerlicher Juristen « gesprochen. Insgesamt sind die Ausführungen wohl nicht so provozierend wie die Zitate verheißen. Wiedergegeben sind hier Eindrücke vom Mainzer Juristentag 1970.

Es war nicht der einzige und nicht der erste Juristentag, an dem der Referent teilgenommen hatte. Von Karlsruhe 1964 bis Wiesbaden 1978 sind es insgesamt sechs gewesen. In Wiesbaden lehnten Vertreter der Wirtschaft gemäßigt fortschrittliche Anträge der arbeitsrechtlichen Abteilung per Stimmkarte und nicht durch Diskussionsargumente ab und sorgten dafür, daß der eher rechtsliberale Rechtsanwalt Konrad Redeker, ein früherer DJT-Präsident, als zu links nicht in das Vertrauensleutegremium gewählt wurde, Vorgänge, die selbst von der konservativen Presse gerügt wurden. ${ }^{2}$ Danach stand es für den Referenten, auch aufgrund früherer Erfahrungen, fest, ein kritischer Jurist sei bei derartigen Veranstaltungen fehl am Platze. Aber der mag für einen anderen, einen Alternativen Juristentag zu haben sein.

\section{Aus der Geschuchte des Deutschen Juristentags}

Vor eıner Antwort, aber auch schon als eine Antwort erscheint eine weitere Befassung mit dem »real existierenden « Juristentag erforderlich. Zum ersten Juristentag wurde $\mathrm{i} 860$ von Männern - Frauen waren ja zu juristischen Professionen wie akademischen überhaupt noch nicht zugelassen - aufgerufen, die, wie Gerhard Dilcher, der Biograph des Juristentags zwischen 1960 und 1980 , schreibt, in den "Idealen des frühen deutschen Liberalismus verwurzelt « waren. ${ }^{3} \mathrm{Zu}$ ihnen gehörte der bekannte Strafrechtslehrer Karl Mittermaier, Mitglied des Pauls-Kirchen-Parla-

\footnotetext{
" Der Beıtrag stellt die überarbeitete Fassung des Eröffnungsreferats beım Alternatıven Jurıstentag vom 23.-25. Nov. 1990 in Hannover dar, ergänzt um Belege. Aus Gründen der Übersıcht sınd solche aus den DJT-Verhandlungen nicht aufgenommen worden. Interessenten werden aufgrund des Inhalts des Textes unschwer un den Verhandlungsprotokollen fündig werden können. - Der Vortragsstil ıst wertgehend beibehalten worden.

Die Veranstalter haben den Referenten offensichtlich nicht wegen seiner Sachkenntnisse ausgesucht, sondern weil er sich schon sest langen Jahren als rechtspolitıscher Kritiker des DJT engagıert hat.

I Vgl. u. a.: Empfiehlt es sıch, den Deutschen Juristentag abzuschaffen? KJ 1970, 473; ders., Jurıstentag: Heerschau des Establishment, eınspruch - die Zeıtung für frese Advokatur, Nr. 28, Februar 1989.

2 Darüber beı Gerhard Dilcher, Der Deutsche Jurıstentag 1960 bis 1980 - Zettgeschichte und Rechtspolitik München, 1980, S. I 3. Die Studie von Dilcher geht über den angegebenen Zeıtabschnıt hınaus und kann als eine zwar wohlwollende, aber nıcht unkritısche Geschichte des DJT insgesamt gewertet werden. Dagegen fast nur rezipierend und affirmativ: Hermann Conrad, Der Deutsche Juristentag 1860-1960, In: Hundert Jahre Deutsches Rechtsleben, Festschrift zum Hundertiährigen Bestehen des Deutschen Juristentages 1860-1960, hisg. von E. v. Caemmerer, E. Friesenhahn, R. Lange, Bd. I, Karlsruhe 1960 , S. $1-36$.

3 Dilcher, S. 8.
} 
ments I 848, wie auch der Obertribunalrat Benedikt Leo Waldeck, i 849 als »Hochverräter « in längere Haft genommen, aber später freigesprochen, ${ }^{4}$ der die Sachvorträge eröffnete, eine Ehre, zu der sich der heutige Juristentag bei einem früheren Häftling gewiß nicht entschließen würde. Der Aufruf zu einem Juristentag »brauste wie ein Donnerhall« durch die deutschen Lande, um eine zeitgemäße Metapher zu verwenden -760 Juristen traten sogleich bei, und 800 waren beim ersten Juristentag anwesend - eine Metapher, die assozieren soll, daß die Gründung auch als nationale Tat verstanden wurde. Liberal und National waren damals noch zwei Seiten einer Medaille; erst nach der Bismarck'schen Reichsgründung wurde das Nationale mehr und mehr eine Sache konservativer Kräfte.

Aber der Juristentag war keine insgeheime Fortsetzung des durchweg von Juristen bestimmten Parlaments von $1848 .^{5}$ Zwar leitete Waldeck seinen Vortrag mit - man möchte sagen - demokratischen Passagen ein, war aber bald beim Fachlichen, bei "Vorfragen betreffend die einheitliche Civilprozeßordnung ", und erst recht verhielten sich so die anderen Referenten. Themen, die in das Politische übergehen könnten, wurden nicht angerührt, auch nicht bei den weiteren Juristentagen. Erst nach fast einem halben Jahrhundert, 1906, wurde ein vorsichtiger Schritt aus dem klassisch juristischen Bereich hinaus mit dem neuen Arbeitsgebiet "Öffentliches Verwaltungsrecht« getan. 1921 kam der völlige Durchbruch mit dem bis dahin peinlich gemiedenen Verfassungsrecht. ${ }^{6}$ Aber sonst wurden durchaus "heiße Eisen « angefaßt. 1860 wurde schon über die Erforderlichkeit der Todesstrafe diskutiert, und bereits I $9 \mathrm{I} 2$ fand sich eine Mehrheit für deren Abschaffung; 195 I eine solche für die Abschaffung der Bestrafung der Homosexualität nach $\$ 175$ StGB. ${ }^{7}$

I 884 hatte der Juristentag schon i 500 Mitglieder, $19123700,{ }^{8}$ prozentual auf die Gesamtheit der Juristen bezogen mehr als heute bei etwa 8500 Mitgliedern. 1933 löste sich der DJT auf und begann 1949 wieder mit 350 Teilnehmern. Eine neue Entwicklung schien sich anzubahnen, auf eine eher elitäre Ausrichtung hin, entsprechend dem Zug der Zeit; der Parlamentarische Rat, ein Honoratiorengremium, hatte sich ja auch gegen massendemokratische Züge der Weimarer Verfassung gewandt. Dilcher beschreibt die ersten Nachkriegsjuristentage als »wissenschaftliche Fachtagungen von hohem Niveau $* .{ }^{9}$ Doch ab 1957 setzte die Zeit der »Heerschauen « ein, mit i 500 Teilnehmern wie auch bei den Juristentagen im folgenden Jahrzehnt. Ab 1970 war eine Teilnehmerzahl von 3000 erreicht, Richtzahl auch für die folgenden Tagungen. 3500 betrug sie in München 1990. ${ }^{10}$

\section{Die Gesellschaft bleibt ausgeklammert}

Der Hauptzweck der DJT-Satzung ( $\$ 2$ in der Fassung v. 27.9. 1988) wie auch die "ständige Übung « richten sich schon seit 1860 auf die "Fortentwicklung des Rechts«. Die Paradigmata haben sich aber in den I 30 Jahren geändert. Damals gab es noch keine Massengesellschaft mit einer allgemeinen Öffentlichkeit. Gesellschaft und Öffentlichkeit wurden von Honoratioren repräsentiert. Die Politiker von 1848 waren keine Funktionäre, sondern Honoratioren und das waren auch die Väter des

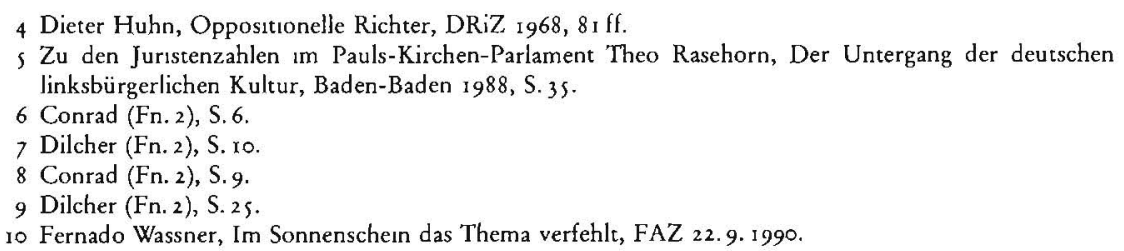


Juristentages. Sie handelten noch in dem Bewußtsein, das Recht bestimme die Gesellschaft, die Rechtseinheit werde auch zur deutschen Einheit führen. Ein Relikt dieser Einstellung findet sich im Abtreibungsurteil des Bundesverfassungsgerichts mit der These von der "sittenbildenden Kraft des Strafrechts. "I Dagegen gilt für heute, was der Rechtssoziologe Eugen Ehrlich schon 1912 ausgesprochen hat: „Der Schwerpunkt der Rechtsentwicklung liegt... weder in der Gesetzgebung noch in der Jurisprudenz oder in der Rechtsprechung, sondern in der Gesellschaft selbst. «"2

Mit dem Verhältnis zur Gesellschaft tut sich der Juristentag schwer. Sie wird in der Satzung nicht angesprochen. Nur von einem »lebendigen Meinungsaustausch unter den deutschen Juristen « ist da die Rede. Vielleicht ist es mehr als ein Zufall, daß dieser Satzungszweck von I 860 bis heute auf die letzte Stelle zurückgefallen ist. Hans-Jochen Vogel kann nicht gefolgt werden, der als Justizminister den Juristentag als eine "große Bürgerinitiative bezeichnet hat. ${ }^{13}$

Aber die Modernität hat auch vor dem Juristentag nicht halt gemacht, indes eine problematische Modernität. Der Juristentag ist heute, so Dilcher, in den Schatten einer Vielzahl machtvoller Verbände getreten, ${ }^{14}$ also auch hier nach Theodor Eschenburg: "Herrschaft der Verbände«. Der DJT hat als Gegenüber keinen auf alle Juristen ausgerichteten Verband wie der Soziologentag den Berufsverband der Soziologen. Es gibt zwar einen Juristinnenbund, aber keinen Verband für alle Juristen. Das gemeinsame Dach bildet allein der Juristentag. Damit ist er überfordert. Das schließt seine Bedeutung als allgemeinen Treffpunkt und Nachrichtenund Informationsbörse nicht aus - so Adolf Arndt, der 1972 verstorbene große Parlamentarier aus den ersten Jahrzehnten des Bundestags und »Kronanwalt der

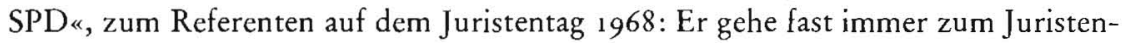
tag, aber nicht wegen der Referate und Diskussionen, sondern um Leute zu treffen und Informationen zu erhalten, wichtig für seine rechtspolitische Arbeit als Politiker. Die negative Seite indes: "Die meisten Teilnehmer sind weder wissenschaftlich, noch rechtspolitisch interessiert. Sie wollen mal den Duft der weiten Wissenschaftswelt schnuppern, sgroße Juristen leibhaftig erleben und alte Kommilitonen wiedersehen. «" Für diese Einschätzung spricht, daß von 3500 Teilnehmern in München sich nur 800 an Abstimmungen beteiligten. ${ }^{16}$

\section{Professionen contra Reformen}

Wie aber steht es mit dem Hauptsatzungszweck, dem Engagement für Reformen? Zwar betonen die Teilnehmer aus den verschiedensten juristischen Professionen und ihre Verbände stets ihre Bereitschaft. Aber es erweist sich bald, daß sie nur für solche Reformen sind, die ihnen nützen, und keineswegs für solche, die von ihnen Opfer erfordern. Um sicher zu gehen, wird jedes Risiko vermieden, weshalb es auch immer wieder zu enttäuschenden Ergebnissen selbst bei solchen Reformansätzen kommt, die auf der Hand liegen und auch von der Öffentlichkeit wie von den Justizministerien unterstützt werden, vor allem bei berufsständischen. ${ }^{17} \mathrm{Zu}$ Wort

II BVerfGE 39, 59.

12 Grundlegung der Soziologie des Rechts, 3. Aufl., Vorrede.

13 Dilcher (Fn. 2), S. 40.

14 Dilcher, S. 99.

15 Rasehorn, Einspruch (Fn. I).

I6 Wassner (Fn. Io).

17 So insbesondere auf dem Manzer Jurıstentag 1970 zum Beamtenrecht (vgl. Rasehorn, KJ (Fn. I)) und zum Anwaltsrecht in München r990, vgl. Jan Albers, Juristentag in München: Thema verfehlt? ZRP 1990,452 . 
melden sich beim Juristentag zumeist jene, denen aufgrund der Rechts- und Tatsachenlage Reformen dringlich erscheinen, darum auch selten Verbandsvertreter, die ihre Funktion zumeist verschweigen und nur die Argumente wiederholen, die sie schon bei der "Anhörung der Verbände" durch das Bundesjustizministerium gebracht haben. Ergibt sich bei den Diskutanten eine starke Mehrheit für Reformen, so sorgen die schweigenden Teilnehmer bei der Abstimmung für die genau entgegengesetzte. ${ }^{18}$

Selbst beı eigentlich »überparteilichen « Gebieten, die nach Reformen "schreien ", erweisen sich die Ergebnisse, wie zuletzt bei der Juristenausbildung, enträuschend. Schwierigkeiten gibt es da bei den Rechtslehrern, kaum noch bei den Praktikern, Schwierigkeiten bei der Beseitigung der Ausuferung in den Nebenfächern bei Studiumsplan und Prüfung. Wenn sich deren Vertreter gegen eine Herabstufung wehren, so wohl nicht aus Professoreneitelkeit, sondern weil es Stellen kosten kann und für den Stelleninhaber Mittel für dessen Institut.

Glatter geht es mit solchen Reformvorschlägen, bei denen die unmıttelbar beteiligten Professionen gewinnen können, und sei es auch nur "Zeit« für Richter, Staatsanwälte und Rechtsanwälte bei dem "Deal « im Strafprozeß (München 1990). Gewinnt aber auch der Außenstehende, der Angeklagte? Noch problematischer kann es bei solchen an sich erfreulichen Reformanliegen wie zum Vormundschaftswesen (Mainz 1988) stehen. Hier hat sich die Mehrheit fortschrittlicher als das Bundesjustizministerium gezeigt, insbesondere im Hinblick auf den Rechtsschutz. Aber auch dahinter steht oft Eigeninteresse: Mehr Stellen für Richter, mehr Honorar für Anwälte. Dafür müssen dann oft Außenstehende zahlen, hier die sozialen Berufe, die mit komplizierteren Gesetzen fertig werden müssen und auch finanziell hintenan stehen, weil die Mittel für die Reformen zunächst an die juristischen Professionen gehen.

Interessen, auch finanzielle, sind in einer pluralistischen Demokratie legitim; aber anstößig ist die schillernde Scheinobjektıvität beim Juristentag, mehr noch, daß Interessen ein Tabu in den hehren Hallen des Rechts bleiben. Wie es schon an der Gesellschaftskritik mangelt, so noch stärker an der Selbstkritik. Darum fällt gar nicht auf, daß eine Institution, die ihrer Tradition und Satzung nach auf den Fortschritt ausgerichtet ist, ihn ausgerechnet mit konservativen Teilnehmern und einer konservativen Organisation erreichen will. Der Jurist ist ja ohnehin, vor allem wegen seiner formalen Ausbildung, wie genug empirische Belege erweisen, auf Beharrung eingestellt. Diese Tendenz wird durch den Einfluß von Verbänden und Institutionen verstärkt, bei denen natürlich etablierte Mitglieder wieder am einflußreichsten sind. Dilcher belegt, daß nicht nur der Prozentzahl, sondern sogar der absoluten Zahl nach weit mehr höhere als »einfache" Richter an Juristentagen teilnehmen. ${ }^{19}$ Je saturierter Verbände und Mitglieder sind, um so mehr sind sie daran interessiert, daß alles so bleibt wie es ist. Um dies zu sichern, beteiligen sie sich überrepräsentativ am Juristentag. Dies wird u. a. über eine Abrechnung als Dienstreise erleichtert; »unabhängige" Juristen verzichten eher wegen der nicht unbeträchtlichen Tagungskosten auf eine Teilnahme. Somit kann das Establishment aus Verbänden und Wirtschaft auf legalem Wege über Stimmkarten erheblichen Einfluß nehmen, ohne daß es ein Sponsern über Spenden bedarf, die nach den Schatzmeisterberichten auch relativ gering sind.

I 8 Es sprıcht nıcht gerade für das krıtısche Urteilsvermögen des, wıe er angibt, ständigen DJT-Teilnehmers Jan Albers, wenn er sıch über die »Zurücklıaltung« der Abstımmungsmehrheıt gegenüber Reformen zur Juristenausbildung und zum Anwaltsrecht erstaunt zeigt.

19 Dilcher (Fn. 2), S. 22. 
Der konservative Einfluß potenziert sıch weiter durch die DJT-Organisation. Nicht alle Mitglieder, sondern nur die am jeweiligen Juristentag teilnehmenden - und das sind wie dargetan die konservativeren - wählen die Organe des Vereins. Anders läßt z. B. die Deutsche Gesellschaft für Soziologie, für die Soziologentage zuständig, die Wahlvorschläge allen Mitgliedern per Post zugehen, die zudem an die Vorschläge nicht gebunden sind. Die teilnehmenden DJT-Mitglieder wählen auch nicht unmittelbar den Vorstand, die Ständige Deputation, so der Traditionsname, sondern seit I955 nur Vertrauensleute, die dann ihrerseits jeweils ein Drittel der 24 Mitglieder neu in die Ständige Deputation wählen. Eine mittelbare Wahl verstärkt erfahrungsgemäß einen konservativen Einfluß, zumal die Ständige Deputatıon Vorschläge für die Wahl der Vertrauensleute vorlegt. Beim letzten Juristentag wurde z. B. für die Berufsgruppe der Rechtsanwälte keinc Frau vorgeschlagen.

Die Ständige Deputation ist der eigentliche Juristentag, während der Tagung selbst und erst recht in der Zwischenzeit. Sie bereitet den Juristentag vor und bestimmt die Beratungsthemen $(\$ 21$ Abs. 2 d. Satz.), wofür indes neuerdings die Mitglieder unverbindliche - Vorschläge machen können. Sie entspricht in der Zusammensetzung dem konservativen Wahlgremium, bemüht sich aber - und muß es auch, um nicht an Einfluß zu verlieren - um eine pluralistische Optik. An- und ausgesprochen wird dies einmal mehr nicht - wohl cine Ausgewogenheit bei den Berufsgruppen $(\$ 7$ d. Satz.). Seit jüngster Zeit gehören ihr - als Alibi - auch einige Frauen und Progressive an. Das Progressive endet mit dem rechten Flügel der SPD. Die rechtspolitischen Interessen von $30 \%$ der Gesellschaft sind also nicht repräsentiert. Jedenfalls in den letzten zwei Jahrzehnten ist kein »echter Linker" mit einem Gutachten oder einem Referat beauftragt worden.

Nicht weniger halbherzig sind die Versuche der Deputation, das gesellschaftspolitische Manko des Juristentags abzubauen. In Sonderveranstaltungen werden zwar aktuelle rechtspolitische Fragen über Podiumsdiskussionen behandelt - so I966 zu NS-Verbrechen, wobei erheblicher innerer Widerstand zu überwinden war, ${ }^{20}$ und jetzt in München zur DDR-Justiz -; die Themen werden aber nur angerissen und nicht umfassend diskutiert. ${ }^{21}$

\section{Ein autoporettsches System?}

Die Diskussion ist überhaupt der große Schwachpunkt. Wie soll sie auch bei mehreren hundert Teilnehmern in den großen Abteilungen realisiert werden können? Es herrscht der Monolog, der der Referenten und anschließend der der Diskutanten. Und selbst zwischen ihnen kommt es nicht zu einer Diskussion, geschweige denn mit dem Auditorium. Uwe Günther: »Nur vorgespielt wird der wissenschaftliche Diskurs, der hinter den Barrikaden vorbereiteter Redebeiträge nicht stattfinden kann. « $^{22}$

Auch geht bei den Themen, wie Dilcher durchblicken $l a ̈ ß t,{ }^{23}$ die "Anstoßwirkung « zugunsten des "Mitfahrermodells" zurück. D. h. der Juristentag springt auf einen Zug auf, den die Öffentlichkeit schon längst besetzt und das Bundesjustizministerium in Fahrt gebracht hat. Gegen die fehlende Aktualität wird eingewandt, es

\footnotetext{
20 Vgl. die ausführliche Darstellung beı Barbara Just-Dahlmann/Helmut Just, Die Gehilfen, Frankfurt/ Man I988, S. $215 \mathrm{ff}$

21 Vgl. Heribert Prantl, Jurıstentag 1990: Thema verfehlt, Süddeutsche Zestung, 18.9. 1990.

22 Leserbinef, enspruch, Nr. 29, Juli 1989.

23 Dilcher (Fn. 2), S. 98 .
} 
werde mindestens der Vorlauf eines Jahres zur Fertigstellung der Gutachten benötigt. ${ }^{24} \mathrm{Da}$ diese inzwischen zu Monographien von oft über 200 Seiten ausgeartet sind, ist tatsächlich ein Jahr knapp bemessen. Aber die Frage ist zu stellen, weshalb man sie gerade für die »Diskussion « beim Juristentag benötigt, sie könnten auch in einem anderen Rahmen, eben als Monographie, verfaßt werden. Kaum $10 \%$ der DJT-Teilnehmer werden sie gelesen haben. Wegen ihrer juristischen Überfracht töten sie die Diskussion eher anstatt sie anzuregen. Als Einstieg reichten gewiß auch die schon ziemlich voluminösen Referate und Korreferate.

Und wie steht es mit dem Output, den Beschlüssen? Hier ist seit dem Mainzer Juristentag 1970 ein völliger Wandel eingetreten. Bis dahin begnügten sich die Abteilungen mit kurzen Tendenzbeschlüssen von 4 bis 5 Punkten. Nunmehr explodierte förmlich der Umfang auf das Zehnfache und mehr. In der Strafrechtsabteilung wurde 1988 insgesamt 77 und in München 65 mal abgestimmt. Es werden dabei Anträge angenommen, die einem 5 Minuten zuvor gefaßten Beschluß widersprechen, weil die Abstimmenden den Überblick verloren hatten, verlieren mußten. So kommt es auch zu fortschrittlichen Passagen in an sich konservativen Beschlüssen. Das große Rätselraten beginnt danach: Was hat der Juristentag beschlossen? Ohnehin: Je mehr beschlossen wird, um so geringer der Einfluß auf die Rechtspolitik. Nochmals Uwe Günther: „Vorgegaukelt wird, die Beschlüsse seien politisch bedeutsam, obwohl ihnen bestenfalls aus Courtoisie Referenz erwiesen wird. «"s

Gleichwohl: Der Juristentag funktioniert, oder besser: blüht und wächst als ein hier mag dieses Modell zutreffen - autopoietisches System, als ein System also, das sich selbst produziert, in sich selbst kreist und die Umwelt nicht mehr benötigt. Mancher mag einwenden, so gesellschaftsfremd sei doch der Juristentag nicht, er sei doch immer - jetzt wird der Referent zynisch - ein gesellschaftliches Ereignis. Das ist er wegen des großartigen Rahmens mit Eröffnungsreden, Empfängen, Theaterbesuchen und Besichtigungen. Vor allem das Damenprogramm hat internationales Niveau. Ingo v. Münch hat 1970 dieses autopoietische System wie folgt persifliert:

"Was würde geschehen, wenn kurz vor oder just in der Zeit der Veranstaltung des Deutschen Juristentages eine umstrittene Grundgesetzänderung im Bundestag beraten, ein wichtiger völkerrechtlicher Vertrag in der Öffentlichkeit diskutıert, eın Justizskandal passieren würde? Nichts. Im Programm nicht vorgesehen, Autobusse zur Sektkellerei schon gemietet. Theaterkarten zurückgelegt. $\aleph^{26}$

\section{Wie kann sich ein Alternativer Juristentag verstehen?}

Das Aufzählen der Defizite des Juristentags gibt Hinweise auf die Struktur eines Alternativen Juristentags. Dabei darf es nicht darum gehen, einem »rechten" Juristentag einen »linken entgegen zu setzen, sondern gerade einen pluralistischen, nach den Worten von Helmut Ridder: einen »anderen Juristentag «, einen mit »erbarmungsloser Selbstkritik «: "Am Ende gar Demokratie «, ${ }^{27}$ einen Juristentag, wie ihn seine Gründer in der heutigen Zeit und Gesellschaft veranstalten würden, einen Juristentag auch, der im vereinten Deutschland den Erfordernissen eines neuen Weges der Rechtspolitik entspricht.

Dafür einige Vorschläge:

I. Mitwirken sollten nicht nur Juristen, sondern auch für Rechtspolitik aufgeschlossene Bürger;

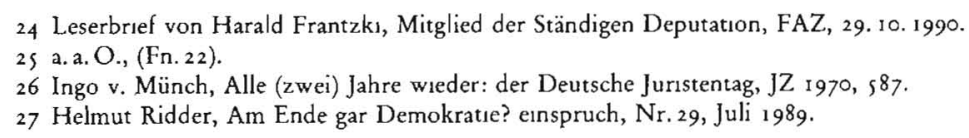


2. Fragen der Rechtskultur und des Rechtsbewußtseins in der Gesellschaft sollten im Mittelpunkt der Diskussion stehen;

3. bei Problemen mit rechtlichem Handlungsbedarf sind Lösungen zu initiieren.

Zu I): Bürgernahe Öffentlichkeit und Transparenz sind entscheidende Kriterien für eine emanzipierte Demokratie. Es wird nicht leicht sein, aufgeschlossene, an Rechtsfragen interessierte Nichtjuristen als Tagungsteilnehmer zu gewinnen. Gewiß werden Soziologen, Politologen und Sozialpädagogen gern mitdiskutieren. Aber die repräsentieren $»$ den « Bürger noch nicht, wobei zunächst Schöffen und ehrenamtliche Richter interessiert werden könnten.

$\mathrm{Zu}$ 2): Themen mit gesellschaftlichem Bezug, die beim Deutschen Juristentag an den Rand gedrängt werden, sollten beim Alternativen im Mittelpunkt stehen. Man mag befürchten, Diskussionen darüber ohne Aufruf an den Gesetzgeber würden zum reinen Selbstzweck, ja zur Selbstbespiegelung. Dagegen ist zu setzen, daß die Diskussion und die damit eingeleitete Bewußtseinsbildung bei den Teilnehmern, also einer größeren $\mathrm{Zahl}$ engagierter Menschen, schon ein Schritt in die Gesellschaft hinein ist. Den Referenten bestärken Erfahrungen von den Soziologentagen, an denen er seit 1974 ständig teilnimmt. Hinzuweisen ist dabei besonders auf den Bamberger Soziologentag (1982), der das Ende der Arbeitsgesellschaft und die Massenarbeitslosigkeit als Dauergefahr prognostiziert hat. Das war kurz nach der "Regierungswende", als die Minister Lambsdorff und Blüm ein Sinken der Arbeitslosenzahl auf wenige Hundertrausende binnen eines Jahres glaubten garantieren zu können. Ein Jahrzehnt Hochkonjunktur läßt erkennen, wie falsch sie lagen und wie wichtig die Prognose der Soziologen für die Grundlagen einer postmodernen Wirtschaftskultur geworden ist.

Die Diskussion über Referate sollte strukturiert werden. Besser wären knappe Statements, um den Unterschied zu den umfassenden, die Diskussion tötenden und wegen ihrer überbetonten Wissenschaftlichkeit den Bürger ausschließenden Gutachten und Referate des Juristentags zu betonen.

$\mathrm{Zu}$ 3): Führt das Nachdenken und Diskutieren zu der Erkenntnis, die Beziehungen zwischen Gesellschaft und Recht seien derart gestört, daß sich ein politischer Handlungsbedarf ergibt, sollte dies jedoch nicht wie beim DJT stets in den Ruf an den Gesetzgeber münden. Es mag reichen und auch erfolgreicher sein, die Gerichte zur Änderung der Rechtsprechung zu bewegen oder über Beiträge zu versuchen, eine herrschende Meinung zu eliminieren sowie auch die allgemeine Öffentlichkeit $\mathrm{zu}$ beeinflussen. Wie mühselig allerdings ein solches Vorgehen ist, weiß der Referent aus jahrzehntelanger Erfahrung auf verschiedenen Ebenen des Rechtswesens. Es gehört eben nach Jacob Burckhardt viel Trommeln dazu, bis die Weltgeschichte einen Schritt vorwärts macht, und zum Fortbewegen der Rechtsgeschichte bedarf es noch viel mehr Trommeln.

Ein Schlußsatz: Wir sind nicht im Jahr 1860 . Die Gründung des Juristentags entsprach dem liberal-demokratischen Zeitgeist der Bildungsschicht und war daher sofort erfolgreich. Der Zeitgeist heute kommt einem konservativen Establishment zugute. Ob er damit auch der Gesellschaft, einer Gesellschaft des Übergangs, zugute kommt, ist zu bezweifeln. Jedenfalls sind Gegenkräfte in der Gesellschaft erforderlich - auf dem Gebiet des Rechtswesens somit ein Alternativer Juristentag. 